\title{
Progress of the MICE experiment
}

\author{
Maurizio Bonesini*i \\ Sezione INFN Milano-Bicocca, Dipartimento di Fisica G. Occhialini, \\ Piazza Scienza 3, 20123 Milano, Italy \\ E-mail: maurizio.bonesini@mib.ch
}

\begin{abstract}
The international Muon Ionization Cooling Experiment (MICE) will perform a systematic investigation of ionization cooling of a muon beam. The demonstration is based on a simplified version of a neutrino factory cooling channel. As the emittance measurement will be done on a particle-by-particle basis, sophisticated beam instrumentation has been developed to measure particle coordinates and timing vs RF. The muon beamline has been characterized and a preliminary measure of the beam emittance, using a particle-by-particle method with only the TOF detector system, has been performed (MICE STEP I). Data taking for the study of the properties that determine the cooling performance (MICE Step IV) has just started in 2015, while the demonstration of ionization cooling with re-acceleration is foreseen for 2017.
\end{abstract}

The European Physical Society Conference on High Energy Physics

22-29 July 2015

Vienna, Austria

\footnotetext{
* Speaker.

${ }^{\dagger}$ on behalf of the MICE Collaboration
} 


\section{Introduction}

A neutrino factory [1] is a muon storage ring with long straight sections, where decaying muons produce collimated, high intensity neutrino beams of defined composition (50\% $v_{e}, 50 \% \bar{v}_{\mu}$ for the $\mu^{+} \rightarrow \bar{v}_{\mu} v_{e} e^{+}$case), with no uncertainties in the spectrum and flux from hadronic production [2]. A neutrino factory will be the most efficient tool to probe the neutrino sector and observe $\mathrm{CP}$ violation in leptons. In addition, at the high energy frontier, colliding muon beams may be a valuable option, benefitting from the use of point-like particles and the much higher mass of a muon with respect to that of the electron [3].

The cooling of muons (accounting for $\sim 20 \%$ of the final costs) will increase the neutrino factory performance and reduce the muon beam emittance up to a factor 2.4 (as described in [4] with a cooling section $75 \mathrm{~m}$ long).

The modest muon cooling needs of a neutrino factory might be traded off by using a larger aperture machine, such as a Fixed-Field Alternating Gradient accelerator [5], but no practical muon collider [6] is conceivable without cooling of at least three orders of magnitude.

Conventional beam cooling methods are ineffective on the short timescale of muon lifetime ( $\tau \sim 2.2 \mu \mathrm{s}$ ). The only effective way is the so-called "ionization cooling" that is accomplished by passing muons through a low- $\mathrm{Z}$ absorber, where they loose energy by ionization and the longitudinal component of momentum is then replenished by RF cavities [7].

The initial goal of the MICE experiment [8] to study a fully engineered cooling cell of the proposed US Study 2 [9] ${ }^{1}$, has been downsized in 2014 to a demonstration of ionization cooling with a simplified lattice based on the available RF cavities and absorber-focus coils (see figure 1).

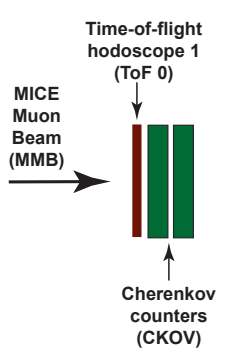

MICE

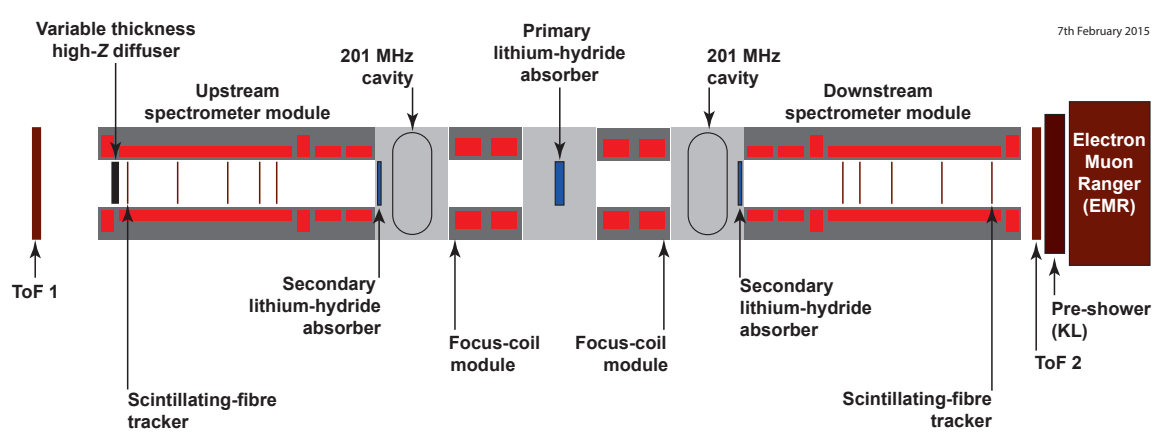

Figure 1: View of the MICE experiment at RAL. The muon beam from ISIS enters from the left. The cooling channel is put between two magnetic spectrometers and two TOF stations (TOF1 and TOF2) to measure particle parameters.

MICE is being done in several steps, of which the first one (STEP I) is the characterization of the beamline.

\section{The MICE STEPI beamline characterization}

The dedicated muon beam from ISIS ${ }^{2}$ enters the MICE cooling section after a $\mathrm{Pb}$ diffuser of

1 a $5.5 \mathrm{~m}$ long cooling section consisting of three liquid hydrogen absorbers and eight $201 \mathrm{MHz}$ RF cavities encircled by lattice solenoids

${ }^{2} 140-240 \mathrm{MeV} / \mathrm{c}$ central momentum, tunable between $3-10 \pi \cdot \mathrm{mm}$ rad input emittance 
adjustable thickness. Pions are produced dipping a hollow titanium cylindrical target into the ISIS proton beam and then captured by a quadrupole triplet (Q1-Q3). The dip depth and timing with respect to the ISIS beam cycle determine the production rate. Muons are then produced from $\pi$ decay inside a $5 \mathrm{~m}$ long superconducting (SC) solenoid (DS) upstream of the first PID detectors.

Particle identification (PID) is obtained upstream of the first tracking solenoid by two TOF stations (TOF0/TOF1) [10] and two threshold Cerenkov counters (CKOVa/CKOVb) [11], that will provide $\pi / \mu$ separation up to $365 \mathrm{MeV} / \mathrm{c}$. A sketch of the present MICE beamline is shown in figure 2.

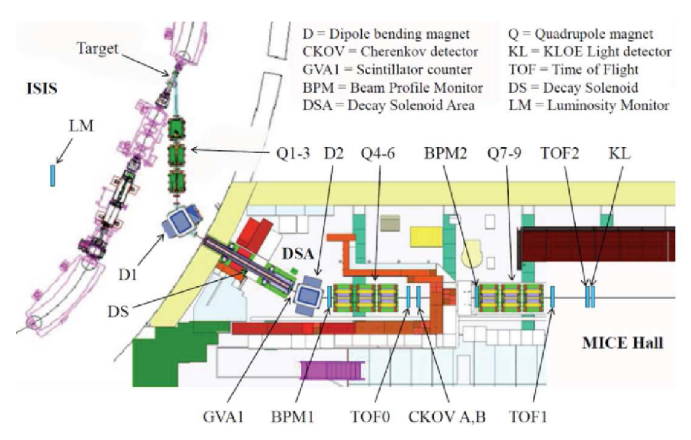

Figure 2: Sketch of the MICE beamline, with installed detectors for STEPI.

Downstream the PID is obtained via an additional TOF station (TOF2) [12] and a calorimeter. All TOF detectors are used to determine the time coordinate $(t)$ in the measurement of the emittance.

A detector resolution $\sim 50 \mathrm{ps}$ is needed for TOF0, to determine the timing with respect to the RF phase to a precision of $5^{0}$. To have a better than $99 \%$ rejection of pions in the incoming muon beam, a resolution $\sim 100 \mathrm{ps}$ for the TOF measurement between TOF0 and TOF1 is needed. All these requirements imply a conservative request of $\sim 50-60 \mathrm{ps}$ for single TOF station resolution. All the TOF stations share a common design based on fast 1 " scintillator counters along the $\mathrm{x} / \mathrm{y}$ directions (to increase measurement redundancy) read at both edges by conventional fast R4998 Hamamatsu photomultipliers ${ }^{3}$.

The downstream calorimeter, made of two separate detectors: KL and EMR, is not intended to be used for energy measurement. Its main goal is to separate muons from decay electrons and undecayed pions. In the MICE calorimeter, EMR determines precisely the muon momentum by range meadurement, while KL acts as an active pre-shower to tag electrons from muon decay. It consists of a Pb-scintillating fiber calorimeter (KL), of the KLOE type [14], with 1-mm diameter blue scintillating fibers glued between $0.3 \mathrm{~mm}$ thick grooved lead plates followed by an electronmuon ranger (EMR), made of a $\sim 1 \mathrm{~m}^{3}$ fully sensitive segmented scintillator block [15].

For MICE STEP IV, the PID detectors have been complemented by two trackers inside the spectrometer solenoids, before and after the cooling section. Each tracker [16] is composed of five stations, each made of three layers of $350 \mu \mathrm{m}$ diameter scintillating fibre doublets, read out by Visible Light Photon Counters (VLPCs).

\footnotetext{
${ }^{3} 1$ " linear focussed PMTs, typical gain $G \sim 5.7 \times 10^{6}$ at B=0 Gauss, risetime $0.7 \mathrm{~ns}, \mathrm{TTS} \sim 160 \mathrm{ps}$. Tests were done in laboratory to characterize them with a dedicated setup [13]
} 
The MICE beamline has been characterized during the so-called STEP I by the use of the TOF system, with data taken mainly in summer 2010.

Figure 3 shows the distribution of the time-of-flight between TOF0 and TOF1 for a low emittance calibration beam (right panel) and a high emittance muon $(\pi \rightarrow \mu)$ beam (left panel). The first peak which is present in both distributions is considered as the time-of-flight of the positrons and is used to determine the absolute value of the time in TOF1. A natural interpretation of the other two peaks is that they are due to forward flying muons from pion decay and pions themselves.
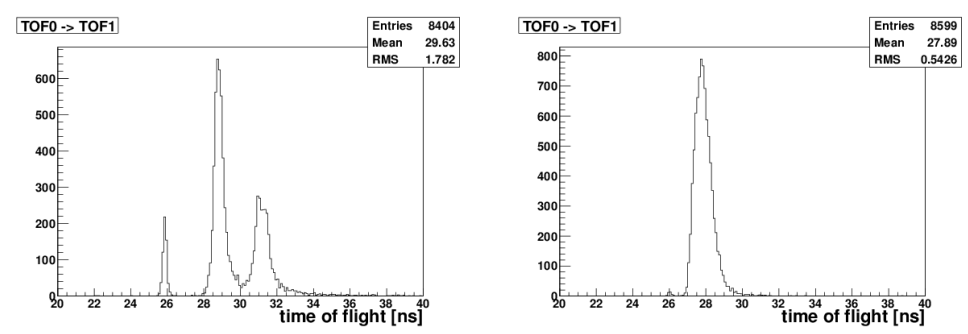

Figure 3: Time of flight between TOF0 and TOF1 for the calibration and muon beams.

Using TOF identification, it was possible to determine the muon rate for the $\pi \rightarrow \mu$ beam as a function of target dip into the ISIS beam (measured as beam loss in $V \cdot m s$ ) [17].

As conventional emittance measurement techniques reach barely a $10 \%$ precision, the final measure of emittance will be done in the MICE beam on a particle-by-particle basis with the trackers (to measure $x, y, x^{\prime}=p_{x} / p_{z}, y^{\prime}=p_{y} / p_{z} . E$ for each particle) and the TOF stations (to measure $t)$. In this way, for an ensemble of $\mathrm{N}\left(\sim 10^{6}\right)$ particles, the input and output emittances may be determined with a precision up to $1 \%$, that allows a sensible extrapolation of the results to the full cooling channel. Due to a schedule delay in the tracking solenoids, in MICE STEPI the emittance was preliminary measured with the TOF system only, deriving from them also the $x, y, x^{\prime}, y^{\prime}$ information for each particle and measuring $p_{z}$ from the time-of-flight between TOF0 and TOF1 [18].

Figure 4 shows these distributions for the MICE baseline beam $\left(\varepsilon=6 \pi \mathrm{mm}\right.$ and $p_{z}=200$ $\mathrm{MeV} / \mathrm{c}$ ) for experimental data and MC simulation. Even if the agreement is not perfect, the beam occupies the desired regions in the trace space. All beams show an RMS beam size of the order of $5-7 \mathrm{~cm}$.

In addition the pion contamination in the MICE muon beam has been measured to be around $1 \%$, as requested for a high precision $(0.1 \%)$ accuracy in the emittance measurement [20].

\section{Further MICE Steps}

A full demonstration of ionization cooling involves a study of the properties that determine the cooling performances and a demonstration of transverse emittance reduction with longitudinal reacceleration. The first item depends on initial beam emittance, muon momentum, absorber material and $\beta_{\perp}$ at the absorber location and its study is the main goal of MICE STEP IV. 

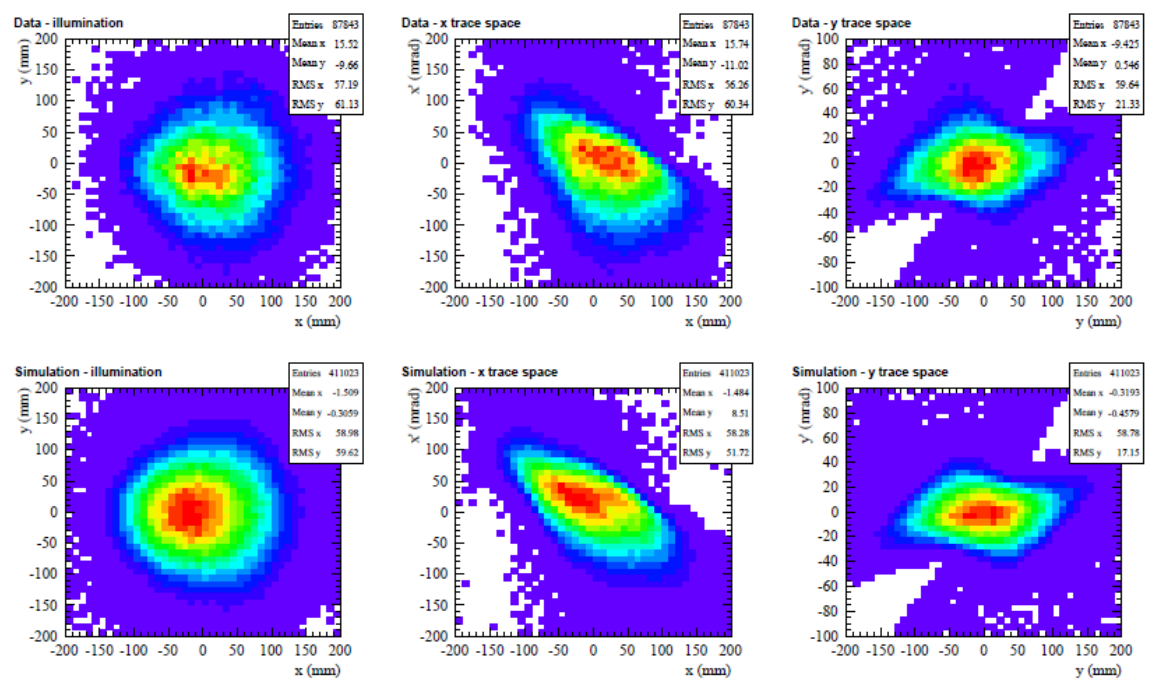

Figure 4: Reconstructed (top) and simulated (bottom) data for the trace plots for the baseline MICE beam $\varepsilon=6 \pi \mathrm{mm}$ and $p_{z}=200 \mathrm{MeV} / \mathrm{c}$.

\subsection{MICE STEP IV}

MICE STEP IV involves an Absorber Focus Coil (AFC) module between two spectrometer solenoids, as shown in figure 5. The AFC module is made of two superconducting (SC) coils
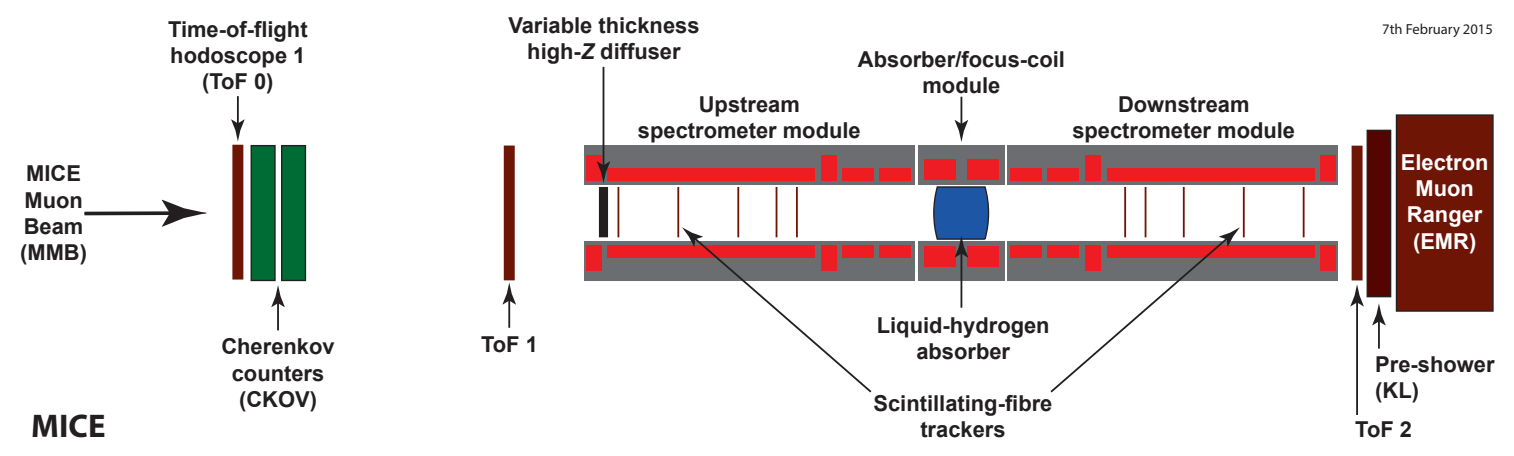

Figure 5: View of the MICE experiment at RAL in the configuration to be used for STEP IV. The muon beam from ISIS enters from the left. The absorber-focus coil module is put between two magnetic spectrometers and two TOF stations (TOF1 and TOF2) that measure particle parameters.

surrounding the absorber. For liquid hydrogen $\left(\mathrm{LH}_{2}\right)$ a cryogenic system is foreseen. A list of possible absorbers is given in table 3.1 .

The lowest equilibrium emittance, corresponding to the optimal cooling channel, is obtained when $\beta_{\perp}$ is minimized and the merit factor $\left(X_{0} \cdot \frac{d E}{d X}\right)$ is maximized. The best merit factor is obtained with liquid hydrogen (a factor two better than the next material: helium). MICE will take data with $\mathrm{LH}_{2}$ and $\mathrm{LiH}$ absorbers, with the possible addition of other materials. In addition to $\mathrm{LiH}$ disks, data taking with $\mathrm{LiH}$ wedge absorbers is foreseen to test longitudinal emittance reduction through emittance exchange [19]. 


\begin{tabular}{|c|c|c|c|}
\hline material & $d E / d X_{\text {Min }}\left(\mathrm{MeVg}^{-1} \mathrm{~cm}^{-2}\right)$ & $X_{0}\left(\mathrm{gcm}^{-2}\right)$ & merit factor \\
\hline $\mathrm{LH} 2$ & 4.034 & 61.28 & 1 \\
$\mathrm{He}$ & 1.937 & 94.32 & 0.74 \\
$\mathrm{LiH}$ & 1.94 & 86.9 & 0.68 \\
$\mathrm{Li}$ & 1.639 & 82.76 & 0.55 \\
$\mathrm{Be}$ & 1.594 & 65.19 & 0.42 \\
\hline
\end{tabular}

Table 1: Comparison of possible absorber materials for ionization cooling.

The two fiber trackers have been recently installed inside the SC solenoids. Each SC solenoid consists of five SC coils on a common aluminium mandrel: three to provide a $4 \mathrm{~T}$ field over the $1 \mathrm{~m}$ long, $20 \mathrm{~cm}$ radius tracking volume with a better than $1 \%$ uniformity and two to match the beam in or out the cooling cell. The two solenoids have been field mapped at RAL and are undergoing training; some data taking for STEP IV has started.

\subsection{The cooling demonstration}

This will be the first engineering demonstration of a "sustainable cooling", as the energy lost in absorbers will be replenished by RF-reacceleration. The initial baseline design with two RF-
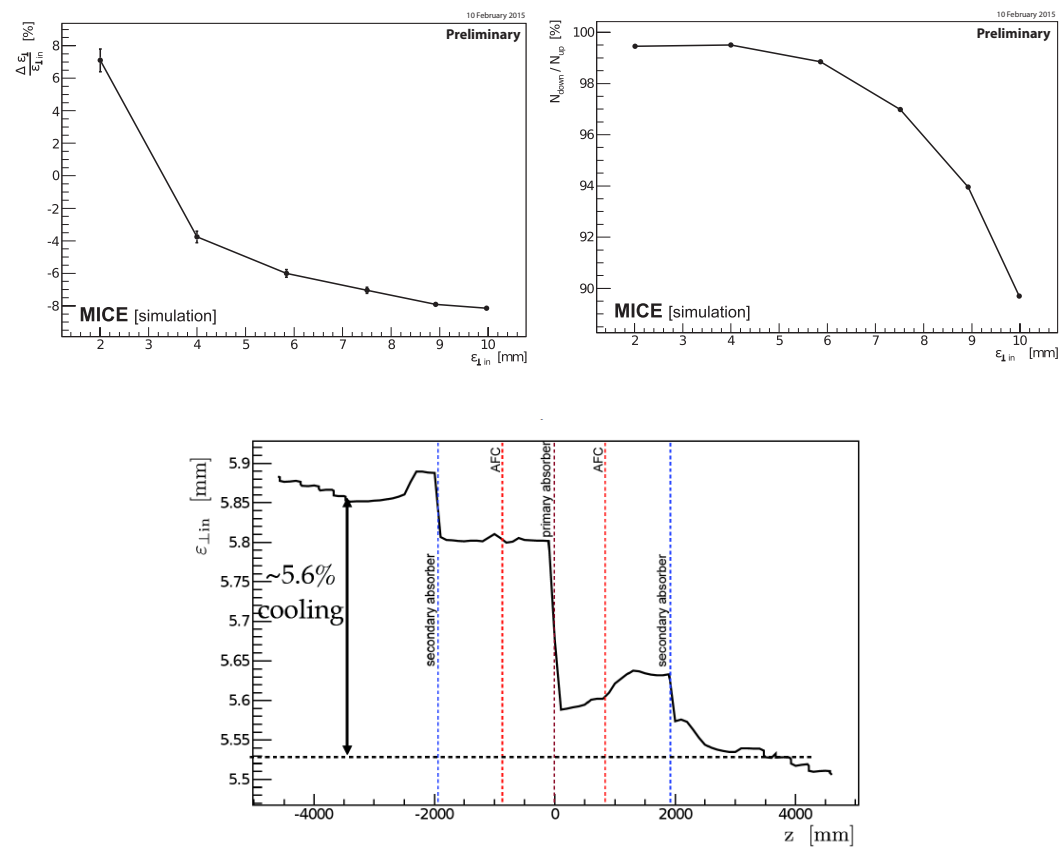

Figure 6: Top-left panel: change in normalised 4D emittance as a function of input emittance. Top-right: beam transmission as function of the input emittance. Bottom panel: evolution of the $4 \mathrm{D}$ emittance in the ionization-colling demo lattice. All plots are for a $6 \pi \cdot \mathrm{mm}, 200 \mathrm{MeV} / \mathrm{c}$ muon beam.

cavity-coupling-coil (RFCC) modules ${ }^{4}$ [9], providing re-acceleration between AFC modules, has been replaced by a simpler design with two single RF cavities, one central (65 $\mathrm{mm}$ ) LiH absorber for cooling and two secondary focus-coil modules for beam focussing, as shown in figure 1. In this

\footnotetext{
${ }^{4}$ each contained four $201 \mathrm{MHz}$ RF cavities and one coupling-coil (CC) solenoid
} 
way, the difficult task of integrating the CC with the RF cavities into a cryostat has been avoided, eliminating a high-risk schedule issue. With this modified setup, a reduction of transverse emittance $\sim 5.6 \%$ with a transmission of $99 \%$ for a $6 \pi \cdot \mathrm{mm}$ input emittance beam (as respect to the baseline $10 \%$ reduction) is expected, as seen in figure 6. Data taking is foreseen to start in 2017.

\section{Conclusions}

The fist step of MICE, devoted to the characterization of the incoming muon beam, has been accomplished, by the construction of the muon beamline and the PID detectors. Obtained detector performances meet the requirements and a preliminary measure of emittance, with TOF only, has been realized. STEP IV for the study of the properties that determine the cooling performance of a low energy muon beam, has just started data taking in mid 2015. This step will involve the complex operation of superconducting magnets of novel design. The next step, the demonstration of ionization cooling with re-acceleration, is scheduled to start in 2017.

\section{References}

[1] Koshkarev, D. G., CERN/ISR-DI/74-62,1974; M. Bogomilov et al., Phys. ReV. ST. Accel. Beams 17 (2014) $12,121002$.

[2] Bonesini, M. and Guglielmi, A., Phys.Rept. 433 (2006),65.

[3] C.M. Ankenbrandt et al., Phys. Rev. ST Accel. Beams 2 (1999) 081001; R.Palmer et al., Nucl. Phys. Proc. Suppl. 51A (1996), 61; R. Palmer et al., Phys. Rev. ST Accel. Beams 8 (2005) 061003.

[4] Choubey S. et al, Int. Design Study for the Neutrino Factory, IDS-NF-20,2011, arXiv:1112.2853.

[5] Rees, G.H. and Kelliher, D.J., Proceedings 46th ICFA Advanced Beam Dynamics Workshop HB2010,2010, p. 54-56; Edgecock R., Int.J.Mod.Phys.A26 (2011) 1736-1743

[6] Geer S., LINAC10 Conference, Tsukuba, Japan, 2010, xarXiv:1202.2140.

[7] A.N. Skrinsky and V.V. Parkhomchuk, Sov. J. Part. Nucl. 12 (1981) 223

[8] G. Gregoire et al. [MICE coll] , MICE Proposal to RAL, 2003.

[9] S.Ozaki et al., BNL-52623, June 2001 ; M.M. Alsharo'a et al., Phys. Rev. ST. Accel. Beams 6,081001 (2003), arXiv:hep-ex/0207031; R. Palmer et al., arXiv:0711.4275.

[10] R. Bertoni et al., Nucl. Instr. and Meth. A615 (2010) 14, arXiv:001.4426.

[11] L. Cremaldi et al., IEEE Trans. Nucl. Sci. 56 (2009) 1475.

[12] R. Bertoni et al., "The construction of the MICE TOF2 detector", MICE-NOTE-DET-286 (2010).

[13] M. Bonesini et al., Nucl. Instr. and Meth. A693 (2012) 130.

[14] A. Aloisio et al. [KLOE coll.], Nucl. Inst. and Meth. A 494 (2002) 326.

[15] D.Lietti et al., Nucl.Instr. and Meth. A604 (2009) 314; R. Asfandiyarov [MICE coll], Nucl. Instr. and Meth. A732 (2013) 451.

[16] M. Ellis et al., Nucl. Instr. and Meth A659 (2011) 136, arXiv:1005.3491.

[17] M. Bogomilov et al. [MICE coll.] , JINST 7 (2012) P05009, arXiv:1203.4089

[18] D. Adams et al. [MICE coll.], Eur. Phys. J. C73 (2013) 2582, arXiv:1306.1509.

[19] M. Bonesini JINST 9 (2014) 01, C01064.

[20] M. Bogomilov et al. [MICE coll.], "Pion contamination in the MICE $\mu$ beam", submitted to JINST. 